\title{
Review Article \\ Oral Janus Kinase Inhibitor for the Treatment of Rheumatoid Arthritis: Tofacitinib
}

\author{
Han Ni, ${ }^{1}$ Soe Moe, ${ }^{2}$ Kay Thi Myint, ${ }^{3}$ and Aung Htet ${ }^{4}$ \\ ${ }^{1}$ Internal Medicine, SEGi University, No. 9 Jalan Teknologi, Taman Sains Selangor, Kota Damansara, PJU 5, \\ 47810 Petaling Jaya, Selangor, Malaysia \\ ${ }^{2}$ Community Medicine, Melaka Manipal Medical College, Jalan Batu Hampar, Bukit Baru, Malacca 75150, Malaysia \\ ${ }^{3}$ Ophthalmology, Melaka Manipal Medical College, Jalan Batu Hampar, Bukit Baru, Malacca 75150, Malaysia \\ ${ }^{4}$ Diagnostic Radiology, Defense Service General Hospital, Naypyitaw, Myanmar
}

Correspondence should be addressed to Han Ni; hanni.dr@gmail.com

Received 30 May 2013; Accepted 29 June 2013

Academic Editors: O. Sander and P. Voulgari

Copyright (C) 2013 Han Ni et al. This is an open access article distributed under the Creative Commons Attribution License, which permits unrestricted use, distribution, and reproduction in any medium, provided the original work is properly cited.

\begin{abstract}
Since the introduction of immune modulators in the treatment of rheumatoid arthritis (RA), there has been hope that orally effective biologic agents would be developed. Tofacitinib, a Janus kinase inhibitor, has become the first oral biologic to receive approval for use in active RA patients. This paper reviews the efficacy and safety profile of Tofacitinib at dosages of $5 \mathrm{mg}$ and $10 \mathrm{mg}$ twice daily. Remarkable improvement in terms of ACR 20 response and HAQ-DI score was noted at month 3 and month 6. DAS 28-4 ESR $<2.6$ achievement was noticeably obvious at month 6 for both dosages. No significant serious adverse events, serious infections, neutropenia, or anaemia were observed compared to placebo. In fact, Tofacitinib $5 \mathrm{mg}$ was even found to have significant protective effect of anaemia in the meta-analysis $(P=0.004)$. Tofacitinib has a noticeable efficacy in controlling disease activity in RA with a manageable safety profile. However, longer studies are needed for its long-term safety profile.
\end{abstract}

\section{Introduction}

Rheumatoid arthritis (RA) is a common immune-mediated systemic disorder, characterized by inflammatory polyarthritis affecting synovium of joints, tendons, and extra-articular sites. It is progressive and leads to joint erosions and deformities, causing premature mortality, functional impairment, and reduced quality of life [1].

The prevalence of RA remains constant at $0.5-1.0 \%$ among various population group $[2,3]$. The prevalence is generally lower in developing countries [4]. In 2005, 1.5 million adults of more than 18 years $(0.6 \%)$ in the United States were estimated to have RA [5]. HLA DRB1 allele is the major genetic risk factor of RA around the world [2].

Conventionally, RA was treated with "Pyramid" approach, where disease modifying antirheumatic drug (DMARD) was deferred until advanced stage. In 1960s and 1970s, gold and penicillamine were the only DMARDs used for RA [6]. However, in 1980s, methotrexate was found to retard or even prevent bone erosions [7]. This has led to dramatic changes in the treatment of RA, with early aggressive use of DMARDs within the first few months of diagnosis, and methotrexate becomes the first line DMARD in RA [6].

In the pathogenesis of rheumatoid arthritis, various inflammatory mediators are found to be involved, among which tumour necrosis factor (TNF) $\alpha$ is the main agent. New drugs targeting these inflammatory mediators have changed the prognosis and outcome of this chronic debilitating disease. Early initiation of DMARDs, either nonbiologic or biologic, has decreased the morbidity of this condition $[8,9]$.

Thus, the recent 2012 American College of Rheumatology (ACR) guidelines on management of RA recommends the use of DMARDs in early RA of less than six months duration as monotherapy for patients with low disease activity and combination therapy for moderate or high disease activity. It also recommends the use of anti-TNF biologics with or without methotrexate for early RA with high disease activity and poor prognostic factors [10]. 


\section{Role of Biologic Agents in Rheumatoid Arthritis}

Interaction between adaptive and innate immune systems is central in the synovial inflammation. In rheumatoid arthritis, the synovium has abundant myeloid cells and plasmacytoid dendritic cells that express cytokines such as interleukin$12,15,18$, and 23 and HLA class II molecules as well as costimulatory molecules that play a role in antigen presentation and T-cell activation [11]. Macrophages are central effectors of synovitis by releasing a variety of inflammatory cytokines, with TNF $\alpha$ and IL- 6 being the most important mediators, ultimately leading to breakdown of extracellular matrix of cartilage and bone [11, 12]. B cells also play a role in autoantigen presentation and cytokine production (e.g., interleukin-6, TNF- $\alpha$, and lymphotoxin- $\beta$ ) [11].

Approved biologic agents used in RA include cytokine inhibitors of TNF alpha (adalimumab, etanercept, infliximab, certolizumab pegol, and golimumab), IL-6 receptor (tocilizumab), IL-1 (anakinra), cell depleting agent targeting CD 20 of B cells (rituximab), and costimulation blocker of cytotoxic T lymphocyte antigen-4 (abatacept) $[11,13,14]$.

However, the limitation of these biologics which requires parenteral administration, either intravenously or subcutaneously, has necessitated the development of new orally effective small molecules for the treatment of rheumatoid arthritis.

\section{JAK Inhibitors/Tofacitinib}

Janus kinase-signal transducer and activator of transcription (JAK-STAT) pathway was first discovered twenty years ago to play an essential role in interferon-dependent cytoplasmic signaling of inflammatory response $[7,25,26]$. JAK family consists of cytosolic tyrosine kinases that regulate cytokinemediated leucocyte maturation and activation, cytokine production, and immunoglobulin production [11, 27, 28]. There are four JAKs: JAK1, JAK2, JAK3, and tyrosine kinase 2 (TYK2) which are selectively associated with the cytoplasmic domains of various cytokine receptors such as IL-2, IL-6, IL-7, IL-12, interferon erythropoietin, and growth hormone $[7,27,29]$. After the ligation of cytokines or growth factors with the respective receptors, JAKs become activated and convey signals to the cytosolic STATs that migrate into the nucleus to promote gene expression [7, 26-29].

JAK3 and TYK2 are primarily involved in immune responses, while JAK1 and JAK2 have various functions ranging from host defense and hematopoiesis to growth and neural development $[26,29]$.

Tofacitinib (CP-690,550) is the most studied JAK inhibitor in RA, which mainly inhibits JAK1 and JAK3 with lesser extent on JAK2. Since JAK2 plays a role in haematopoiesis, this selectivity is advantageous for Tofacitinib with lesser haematological adverse effects $[7,29]$. In animal models of experimental arthritis, Tofacitinib was shown to be effective in preventing cartilage damage [7]. Subsequent studies done in human RA patients also reveal promising results with improvement in disease activity. In this review, we will analyze the efficacy and safety of Tofacitinib in active rheumatoid arthritis patients.

\section{Material and Methodology}

We identified published randomized controlled trials on Tofacitinib in RA patients by searching the PubMed database (until May 2013) using the search terms "Tofacitinib" OR "Janus Kinase inhibitor" AND "Rheumatoid arthritis" AND "randomiz/sed controlled trial." We also searched the reference lists of all included studies, US Food and Drug Administration (FDA) [30], pharmaceutical websites (Pfizer), and proceedings and abstracts of rheumatology conferences. We included studies of adult RA patients (18 years or older) diagnosed by the ACR 1987 revised criteria (Table 4). We only included the active disease defined as the presence of 6 or more tender or painful joints (out of 68 specific joints examined) and 6 or more swollen joints (out of 66 specific joints examined) and had either Westergren erythrocyte sedimentation rate (ESR) of more than $28 \mathrm{~mm}$ per hour or C-reactive protein (CRP) level of more than $7 \mathrm{mg}$ per liter. These patients are either methotrexate/DMARD naive or with incomplete response to prior biologic or nonbiologic DMARDs who continue taking methotrexate, NSAIDs, or steroids at the stable dosage. We excluded studies with other kinase inhibitors or oral biologic agents. We analyzed the ACR 20 response at month 3 or month 6; Disease Activity score for 28 -joint counts based on the erythrocyte sedimentation rate (DAS 28-4 ESR) $<2.6$ at month 3 or month 6; change in Health Assessment Questionnaire-Disability Index (HAQ-DI) score from baseline at month 3 for the efficacy of Tofacitinib; serious adverse events, serious infections, and cytopenia for safety. ACR 20 response is defined as at least $20 \%$ improvement in both swollen and tender joint counts and three out of the following five variables: patient and evaluator global disease activity, pain assessment, functional disability, and acute-phase reactants (sedimentation rate or C-reactive protein).

\section{Data Extraction and Management}

The review authors assessed the search results according to the eligibility criteria for inclusion in this review and extracted data from the included trials. The data were entered into the Review Manager Software version 5.2 for statistical analysis. Mantel-Haenszel odds ratio (OR) with 95\% confidence interval (CI) is used for meta-analysis of dichotomous outcome data.

\section{Results}

6.1. Description of Studies. Our search strategy yielded 14 potentially relevant results, among which nine (four phase 3 trials $[15,16,18,19]$ and five phase 2 trials [20-24]) fulfill our selection criteria. Further search from the conference proceedings indentified the abstract of the fifth phase 3 trial [17]. A total of ten RCTs met our inclusion criteria (Table 1), 
TABLE 1: Summary of published studies on Tofacitinib.

\begin{tabular}{|c|c|c|c|c|c|}
\hline Study & Phase & Duration & Participants & Intervention & Primary Outcome \\
\hline $\begin{array}{l}\text { Burmester et } \\
\text { al. [15] }\end{array}$ & 3 & 6 months & $\begin{array}{l}\text { Moderate to severe RA Patients } \\
\text { with inadequate response to } \\
\text { TNF } \alpha \text { inhibitors }(N=399)\end{array}$ & $\begin{array}{l}\text { Tofacitinib } 5 \mathrm{mg} \text { bd; } \\
\text { Tofacitinib } 10 \mathrm{mg} \text { bd; } \\
\text { placebo all with methotrexate }\end{array}$ & $\begin{array}{l}\text { ACR } 20 \text { response at month } 3 \text {; } \\
\text { DAS } 28-4 \text { ESR }<2.6 \text { at month } 3 \text {; } \\
\text { HAQ-DI at month } 3 \text { (change } \\
\text { from baseline) }\end{array}$ \\
\hline $\begin{array}{l}\text { Fleischmann } \\
\text { et al. [16] }\end{array}$ & 3 & 6 months & $\begin{array}{l}\text { Active RA patients receiving } \\
\text { stable doses of antimalarial } \\
\text { agents } \\
\text { Prior inadequate response to at } \\
\text { least one DMARD (biologic or } \\
\text { nonbiologic) }(N=611)\end{array}$ & $\begin{array}{l}\text { Tofacitinib } 5 \mathrm{mg} \text { bd; } \\
\text { Tofacitinib } 10 \mathrm{mg} \text { bd; } \\
\text { placebo for } 3 \mathrm{months} \text { followed by } \\
\text { Tofacitinib } 5 \mathrm{mg} \text { bd; } \\
\text { placebo for } 3 \text { months followed by } \\
\text { Tofacitinib } 10 \mathrm{mg} \text { bd }\end{array}$ & $\begin{array}{l}\text { ACR } 20 \text { response at month } 3 \text {; } \\
\text { DAS } 28-4 \text { ESR }<2.6 \text { at month } 3 \text {; } \\
\text { HAQ-DI at month } 3 \text { (change } \\
\text { from baseline) }\end{array}$ \\
\hline $\begin{array}{l}\text { Kremer et al. } \\
{[17]}\end{array}$ & 3 & 12 months & $\begin{array}{l}\text { Active RA Patients with } \\
\text { inadequate response to } \geq 1 \\
\text { DMARD }(N=792)\end{array}$ & $\begin{array}{l}\text { Tofacitinib } 5 \mathrm{mg} \text { bd; } \\
\text { Tofacitinib } 10 \mathrm{mg} \text { bd; } \\
\text { placebo }\end{array}$ & $\begin{array}{l}\text { ACR } 20 \text { response at month } 6 \text {; } \\
\text { DAS } 28-4 \text { ESR < } 2.6 \text { at month } 6 \text {; } \\
\text { HAQ-DI at month } 3 \text { (change } \\
\text { from baseline) }\end{array}$ \\
\hline $\begin{array}{l}\text { van der } \\
\text { Heijde et al. } \\
{[18]}\end{array}$ & 3 & 24 months & $\begin{array}{l}\text { Active RA patients receiving } \\
\text { background methotrexate } \\
(N=797)\end{array}$ & $\begin{array}{l}\text { Tofacitinib } 5 \mathrm{mg} \text { bd; } \\
\text { Tofacitinib } 10 \mathrm{mg} \text { bd; } \\
\text { placebo followed by Tofacitinib } \\
5 \mathrm{mg} \text { bd; } \\
\text { placebo followed by Tofacitinib } \\
10 \mathrm{mg} \text { bd }\end{array}$ & $\begin{array}{l}\text { ACR } 20 \text { response at month } 6 \text {; } \\
\text { DAS } 28-4 \text { ESR < } 2.6 \text { at month 6; } \\
\text { HAQ-DI at month } 3 \text { (change } \\
\text { from baseline) }\end{array}$ \\
\hline $\begin{array}{l}\text { van } \\
\text { Vollenhoven } \\
\text { et al. [19] }\end{array}$ & 3 & 12 months & $\begin{array}{l}\text { Active RA patients receiving } \\
\text { stable doses of methotrexate } \\
(N=717)\end{array}$ & $\begin{array}{l}\text { Tofacitinib } 5 \mathrm{mg} \text { bd; } \\
\text { Tofacitinib } 10 \mathrm{mg} \text { bd; } \\
\text { adalimumab } 40 \mathrm{mg} \text { every } 2 \\
\text { weeks; } \\
\text { placebo }\end{array}$ & $\begin{array}{l}\text { ACR } 20 \text { response at month 6; } \\
\text { DAS } 28-4 \text { ESR }<2.6 \text { at month 6; } \\
\text { HAQ-DI at month } 3 \text { (change } \\
\text { from baseline) }\end{array}$ \\
\hline
\end{tabular}

placebo

Tofacitinib $1 \mathrm{mg}, 3 \mathrm{mg}, 5 \mathrm{mg}$,

$10 \mathrm{mg}$, or $15 \mathrm{mg}$ bd;

$\begin{array}{llll}\begin{array}{l}\text { Fleischmann } \\ \text { et al. [20] }\end{array} & \text { 2b } \quad 24 \text { weeks } & \begin{array}{l}\text { Active RA Patients with } \\ \text { inadequate response to DMARD }\end{array}\end{array}$

adalimumab $40 \mathrm{mg}$ every 2

weeks for 6 injections followed

ACR 20 response at week 12

by Tofacitinib $5 \mathrm{mg}$ bd;

placebo

\begin{tabular}{|c|c|c|c|c|c|}
\hline $\begin{array}{l}\text { Kremer et al. } \\
{[21]}\end{array}$ & $2 b$ & 24 weeks & $\begin{array}{l}\text { Active RA patients receiving } \\
\text { stable doses of methotrexate with } \\
\text { inadequate response to } \\
\text { methotrexate alone }(N=507)\end{array}$ & $\begin{array}{l}\text { Tofacitinib } 20 \mathrm{mg} \text { daily; } \\
\text { Tofacitinib } 1 \mathrm{mg}, 3 \mathrm{mg}, 5 \mathrm{mg} \text {, } \\
10 \mathrm{mg} \text {, or } 15 \mathrm{mg} \text { bd; } \\
\text { placebo }\end{array}$ & ACR 20 response at week 12 \\
\hline $\begin{array}{l}\text { Tanaka et al. } \\
{[22]}\end{array}$ & 2 & 12 weeks & $\begin{array}{l}\text { Active RA patients receiving } \\
\text { stable doses of methotrexate with } \\
\text { inadequate response to } \\
\text { methotrexate alone }(N=140)\end{array}$ & $\begin{array}{l}\text { Tofacitinib } 1 \mathrm{mg}, 3 \mathrm{mg}, 5 \mathrm{mg} \text {, and } \\
10 \mathrm{mg} \text { bd; } \\
\text { placebo }\end{array}$ & ACR 20 response at week 12 \\
\hline $\begin{array}{l}\text { Kremer et al. } \\
{[23]}\end{array}$ & $2 \mathrm{a}$ & 6 weeks & $\begin{array}{l}\text { Active RA Patients with } \\
\text { inadequate or toxic response to } \\
\text { methotrexate, etanercept, } \\
\text { infliximab, or adalimumab } \\
(N=264)\end{array}$ & $\begin{array}{l}\text { Tofacitinib } 5 \mathrm{mg}, 15 \mathrm{mg} \text {, and } \\
30 \mathrm{mg} \text { bd; } \\
\text { placebo }\end{array}$ & ACR 20 response at week 6 \\
\hline $\begin{array}{l}\text { Coombs et al. } \\
{[24]}\end{array}$ & 2 & 6 weeks & $\begin{array}{l}\text { Moderate to severe active RA } \\
\text { Patients with inadequate } \\
\text { response to methotrexate or a } \\
\text { TNF } \alpha \text { inhibitor }(N=264)\end{array}$ & $\begin{array}{l}\text { Tofacitinib } 5 \mathrm{mg}, 15 \mathrm{mg} \text {, and } \\
30 \mathrm{mg} \text { bd; } \\
\text { placebo }\end{array}$ & $\begin{array}{l}\text { Visual analogue scale scores at } \\
\text { week 6; } \\
\text { HAQ-DI (change from baseline) } \\
\text { and short form-36 (SF-36) at } \\
\text { week } 6\end{array}$ \\
\hline
\end{tabular}


TABLE 2: Change in HAQ-DI score at month 3 (from baseline).

\begin{tabular}{|c|c|c|c|c|c|}
\hline Study & Total $(N)$ & Tofacitinib $5 \mathrm{mg}$ bd & Tofacitinib $10 \mathrm{mg}$ bd & Placebo & Adalimumab $40 \mathrm{mg}$ every 2 weeks \\
\hline Burmester et al. [15] & 399 & $-0.43^{\S}$ & $-0.46^{\S}$ & -0.18 & - \\
\hline Fleischmann et al. [16] & 611 & $-0.50^{* *}$ & $-0.57^{* *}$ & -0.19 & - \\
\hline Kremer et al. [17] & 792 & $-0.46^{\S}$ & $-0.56^{\S}$ & -0.21 & - \\
\hline van der Heijde et al. [18] & 797 & $-0.40^{\#}$ & $-0.54^{\S}$ & -0.15 & - \\
\hline van Vollenhoven et al. [19] & 717 & $-0.55^{* *}$ & $-0.61^{* *}$ & -0.24 & $-0.49^{* *}$ \\
\hline Fleischmann et al. [20] & 384 & $-0.51^{*}$ & $-0.66^{\S}$ & -0.25 & - \\
\hline Kremer et al. [21] & 507 & $-0.49^{* *}$ & $-0.39^{*}$ & -0.16 & - \\
\hline Tanaka et al. [22] & 140 & $-0.49^{* *}$ & $-0.57^{* *}$ & -0.05 & - \\
\hline
\end{tabular}

${ }^{*}$ Significant $P<0.05 ;{ }^{* *}$ significant $P<0.001 ;{ }^{\S}$ significant $P<0.0001$; ${ }^{\#}$ significance not declared.

TABLE 3: Analysis of safety profile of Tofacitinib.

\begin{tabular}{|c|c|c|c|c|}
\hline Outcome & Number of studies & $\begin{array}{l}\text { Number of } \\
\text { participants }\end{array}$ & $\begin{array}{c}\text { Odds ratio } \\
\text { [Confidence Interval] }\end{array}$ & $P$-value \\
\hline $\begin{array}{l}\text { Serious adverse events with Tofacitinib } 5 \mathrm{mg} \text { versus placebo at month } \\
0-3\end{array}$ & $5[15-19]$ & 1891 & $0.80[0.47,1.35]$ & 0.39 \\
\hline $\begin{array}{l}\text { Serious adverse events with Tofacitinib } 10 \mathrm{mg} \text { versus placebo at month } \\
0-3\end{array}$ & 5 [15-19] & 1896 & $0.77[0.45,1.31]$ & 0.33 \\
\hline Serious infections with Tofacitinib $5 \mathrm{mg}$ versus placebo at month $0-3$ & $4[15,16,18,19]$ & 1423 & $1.91[0.31,11.70]$ & 0.49 \\
\hline Serious infections with Tofacitinib $10 \mathrm{mg}$ versus placebo at month $0-3$ & $4[15,16,18,19]$ & 1418 & $2.10[0.44,9.94]$ & 0.35 \\
\hline Serious adverse events with Tofacitinib $5 \mathrm{mg}$ versus $10 \mathrm{mg}$ at month 3-6 & $5[15-19]$ & 2427 & $1.28[0.80,2.03]$ & 0.30 \\
\hline Serious adverse events with Tofacitinib $5 \mathrm{mg}$ versus $10 \mathrm{mg}$ at month 6-12 & $2[18,19]$ & 1042 & $1.53[0.79,2.97]$ & 0.21 \\
\hline Serious infections with Tofacitinib $5 \mathrm{mg}$ versus $10 \mathrm{mg}$ at month 3-6 & $4[15,16,18,19]$ & 1797 & $1.62[0.67,3.94]$ & 0.28 \\
\hline Serious infections with Tofacitinib $5 \mathrm{mg}$ versus $10 \mathrm{mg}$ at month 6-12 & $2[18,19]$ & 1042 & $0.74[0.16,3.31]$ & 0.69 \\
\hline $\begin{array}{l}\text { Mild neutropenia }\left(1500-1999 \text { cells } / \mathrm{mm}^{3}\right) \text { with Tofacitinib } 5 \mathrm{mg} \text { versus } \\
\text { placebo at month } 0-3\end{array}$ & $4[15,16,18,19]$ & 1308 & $1.55[0.64,3.77]$ & 0.33 \\
\hline $\begin{array}{l}\text { Mild neutropenia }\left(1500-1999 \text { cells } / \mathrm{mm}^{3}\right) \text { with Tofacitinib } 10 \mathrm{mg} \text { versus } \\
\text { placebo at month } 0-3\end{array}$ & $4[15,16,18,19]$ & 1312 & $1.97[0.83,4.67]$ & 0.12 \\
\hline $\begin{array}{l}\text { Moderate to severe neutropenia }\left(500-1499 \text { cells } / \mathrm{mm}^{3}\right) \text { with Tofacitinib } \\
5 \mathrm{mg} \text { versus placebo at month } 0-3\end{array}$ & $4[15,16,18,19]$ & 1308 & $3.26[0.71,14.95]$ & 0.13 \\
\hline $\begin{array}{l}\text { Moderate to severe neutropenia }\left(500-1499 \text { cells } / \mathrm{mm}^{3}\right) \text { with Tofacitinib } \\
10 \mathrm{mg} \text { versus placebo at month } 0-3\end{array}$ & $4[15,16,18,19]$ & 1312 & $2.99[0.52,17.02]$ & 0.22 \\
\hline $\begin{array}{l}\text { Anaemia (decreased haemoglobin }-1 \text { to }-3 \mathrm{~g} / \mathrm{dL} \text { ) with Tofacitinib } 5 \mathrm{mg} \\
\text { versus placebo at month } 0-3\end{array}$ & $4[15,16,18,19]$ & 1339 & $0.56[0.38,0.83]$ & $0.004^{*}$ \\
\hline $\begin{array}{l}\text { Anaemia (decreased haemoglobin }-1 \text { to }-3 \mathrm{~g} / \mathrm{dL} \text { ) with Tofacitinib } 10 \mathrm{mg} \\
\text { versus placebo at month } 0-3\end{array}$ & $4[15,16,18,19]$ & 1337 & $1.05[0.74,1.48]$ & 0.80 \\
\hline
\end{tabular}

${ }^{*}$ Significant.

but two of them were not involved in the data analysis because their outcome measures did not meet our criteria. Both of them were only six-week studies $[23,24]$.

6.2. Efficacy of Tofacitinib. Meta-analysis of the efficacy outcome measures showed that the ACR 20 response at month 3 and month 6 was significantly better with Tofacitinib at both $5 \mathrm{mg}$ and $10 \mathrm{mg}$ twice daily compared to placebo (Figures $1(\mathrm{a})-1(\mathrm{~d}))$. The proportion of active RA patients whose DAS 28-4 ESR < 2.6 at month 6 was significantly higher for Tofacitinib at both $5 \mathrm{mg}$ and $10 \mathrm{mg}$. However, at month 3, DAS 28-4 ESR $<2.6$ achievement was not significant with $5 \mathrm{mg}$ dosage while it was significant for $10 \mathrm{mg}$ twice daily dosage compared with placebo (Figures 1(e)-1(h)).
Consistent and statistically significant improvement in HAQ-DI score from the baseline at month 3 with Tofacitinib $5 \mathrm{mg}$ and $10 \mathrm{mg}$ compared to placebo was noted across all the included studies (Table 2).

6.3. Safety of Tofacitinib. No statistically significant difference was noted for safety outcome measures with Tofacitinib $5 \mathrm{mg}$ or $10 \mathrm{mg}$ at month 3 and 6 compared to placebo (Table 3 ). There were no reported cases of life-threatening neutropenia in all the included phase 3 studies [15-19]. Occurrence of both mild neutropenia and moderate to severe neutropenia were not statistically higher among the Tofacitinib group.

Interestingly, Tofacitinib $5 \mathrm{mg}$ twice daily was found to have a significant protective effect compared to placebo 


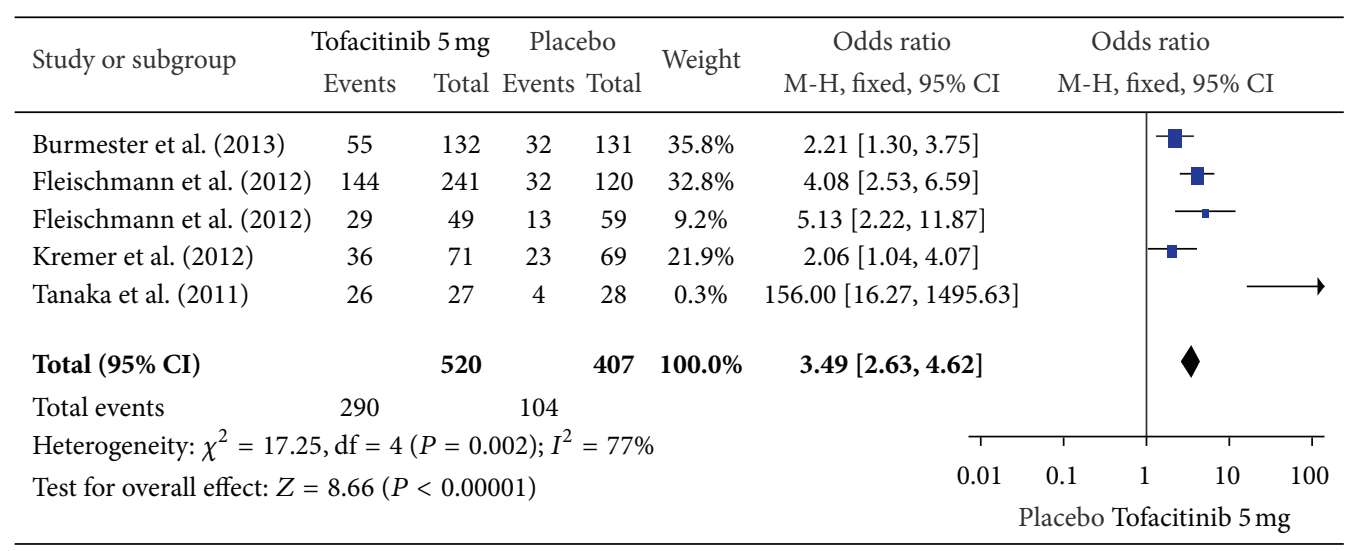

(a) ACR 20 response at month 3 (Tofacitinib $5 \mathrm{mg}$ )

\begin{tabular}{|c|c|c|c|c|c|c|c|c|c|}
\hline \multirow{2}{*}{$\begin{array}{l}\text { Study or subgroup } \\
\text { Fleischmann et al. (2012) }\end{array}$} & \multicolumn{2}{|c|}{ Tofacitinib $5 \mathrm{mg}$} & \multicolumn{2}{|c|}{$\begin{array}{c}\text { Placebo } \\
\text { Events Total }\end{array}$} & Weight & $\begin{array}{c}\text { Odds ratio } \\
\text { M-H, fixed, 95\% CI }\end{array}$ & \multicolumn{2}{|c|}{$\begin{array}{c}\text { Odds ratio } \\
\text { M-H, fixed, 95\% CI }\end{array}$} & \\
\hline & 25 & 49 & 15 & 59 & $8.2 \%$ & $3.06[1.36,6.87]$ & & \multicolumn{2}{|l|}{$\longrightarrow$} \\
\hline Kremer et al. (2011) & 164 & 311 & 49 & 157 & $37.7 \%$ & $2.46[1.64,3.68]$ & & \multicolumn{2}{|l|}{ - } \\
\hline van der Heijde et al. (2013) & 159 & 309 & 39 & 154 & $31.0 \%$ & $3.13[2.04,4.79]$ & & \multicolumn{2}{|l|}{-} \\
\hline van Vollenhoven et al. (2012) & 2) 101 & 196 & 30 & 106 & $23.1 \%$ & $2.69[1.62,4.47]$ & & \multicolumn{2}{|l|}{-} \\
\hline Total $(95 \% \mathrm{CI})$ & & 865 & & 476 & $100.0 \%$ & $2.77[2.17,3.53]$ & & \multicolumn{2}{|l|}{ 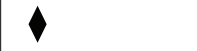 } \\
\hline Total events & \multicolumn{2}{|l|}{449} & \multicolumn{3}{|l|}{133} & & & \multirow{2}{*}{\multicolumn{2}{|c|}{\begin{tabular}{|lll} 
& & \\
1 & 10 & 100
\end{tabular}}} \\
\hline Heterogeneity: $\chi^{2}=0.71, \mathrm{df}=$ & $\mathrm{f}=3(P$ & $=0.87)$ & $; I^{2}=0$ & & & 0.01 & 0.1 & & \\
\hline \multicolumn{6}{|c|}{ Test for overall effect: $Z=8.24(P<0.00001)$} & \multicolumn{4}{|c|}{ Placebo Tofacitinib $5 \mathrm{mg}$} \\
\hline
\end{tabular}

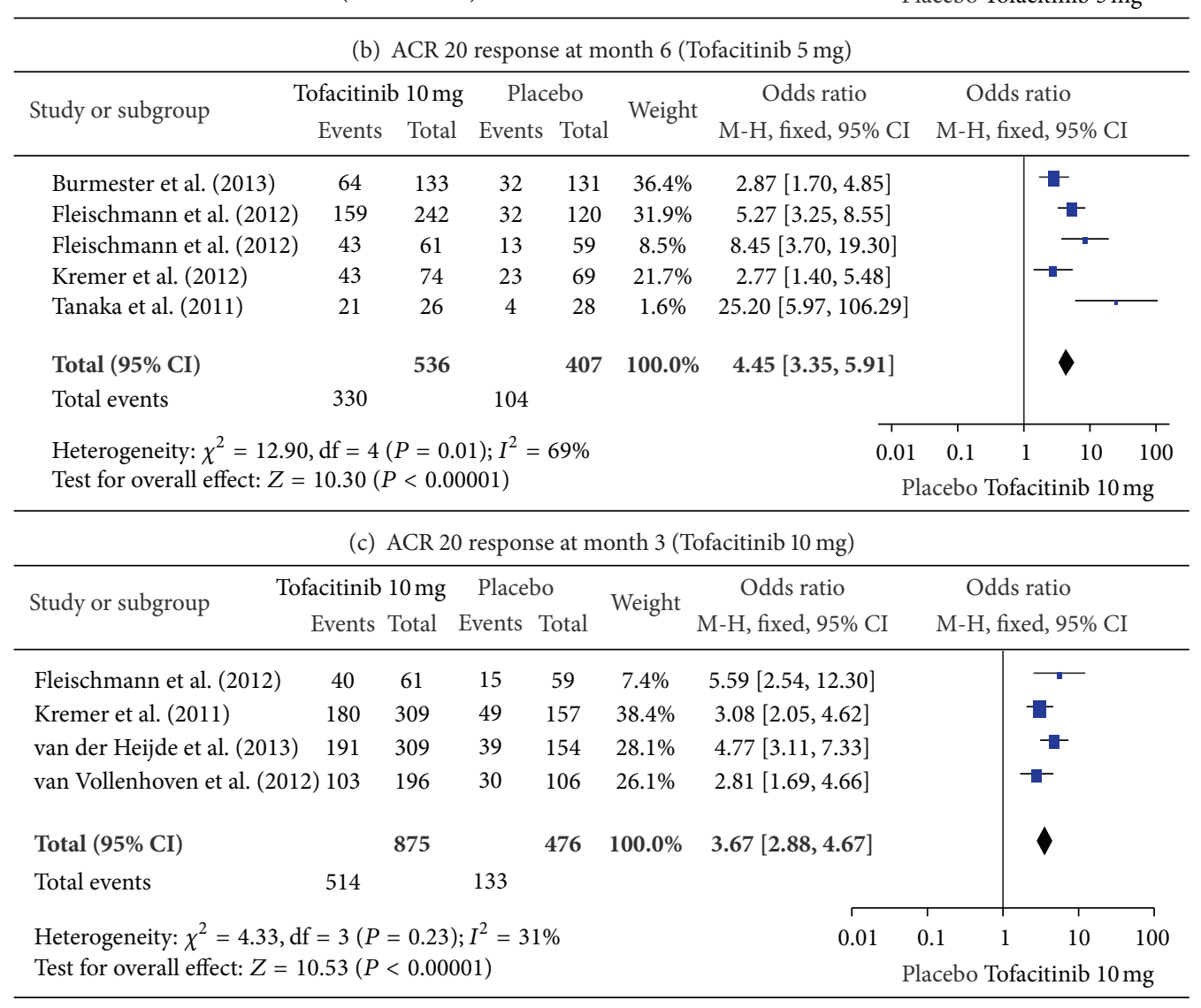

(d) ACR 20 response at month 6 (Tofacitinib $10 \mathrm{mg}$ )

Figure 1: Continued. 


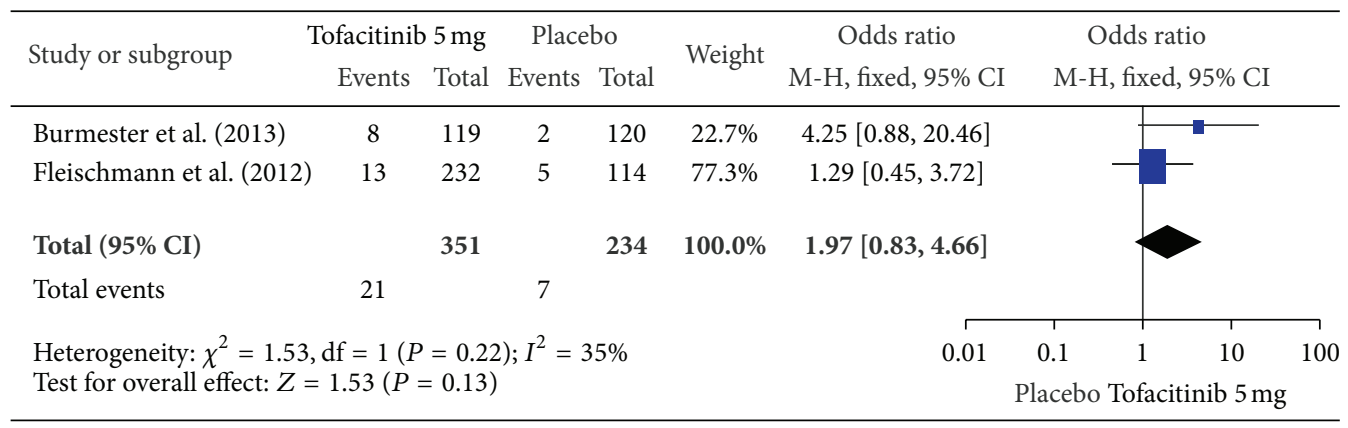

(e) DAS 28-4 ESR $<2.6$ at month 3 (Tofacitinib $5 \mathrm{mg}$ )

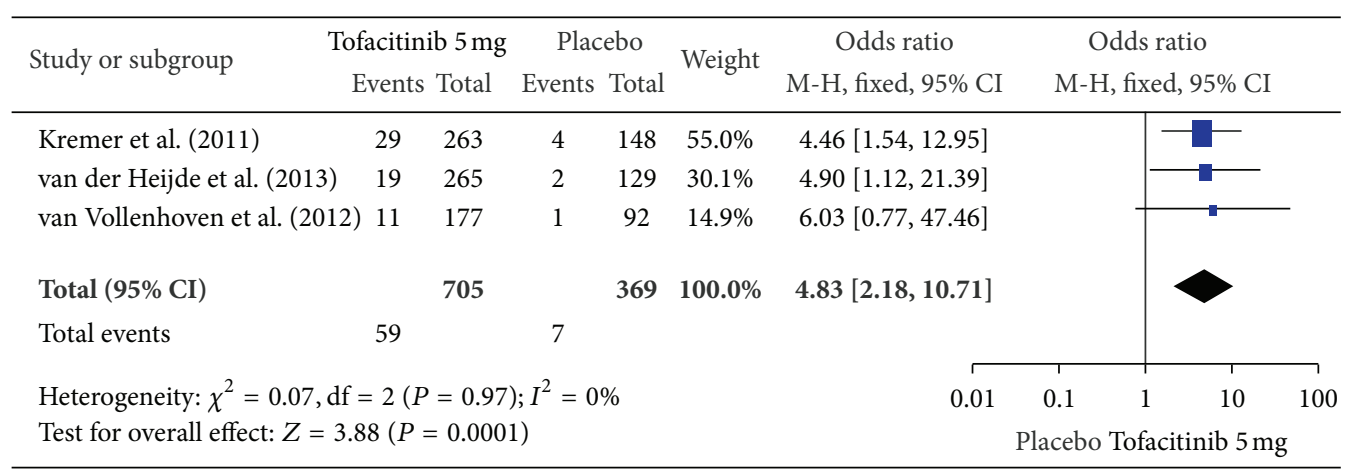

(f) DAS 28-4 ESR $<2.6$ at month 6 (Tofacitinib $5 \mathrm{mg}$ )

\begin{tabular}{|c|c|c|c|c|c|c|c|c|c|}
\hline \multirow{2}{*}{ Study or subgroup } & \multicolumn{2}{|c|}{ Tofacitinib $10 \mathrm{mg}$} & \multicolumn{2}{|c|}{ Placebo } & \multirow{2}{*}{ Weight } & \multirow{2}{*}{$\begin{array}{c}\text { Odds ratio } \\
\text { M-H, fixed, 95\% CI }\end{array}$} & \multirow{2}{*}{\multicolumn{2}{|c|}{$\begin{array}{c}\text { Odds ratio } \\
\text { M-H, fixed, 95\% CI }\end{array}$}} & \\
\hline & Events & Total & Events & Total & & & & & \\
\hline Burmester et al. (2013) & 11 & 125 & 2 & 120 & $23.4 \%$ & $5.69[1.23,26.25]$ & & - & \\
\hline Fleischmann et al. (2012) & 20 & 229 & 5 & 114 & $76.6 \%$ & $2.09[0.76,5.71]$ & & & \\
\hline Total (95\% CI) & & 354 & & 234 & $100.0 \%$ & $2.93[1.27,6.74]$ & & & \\
\hline Total events & 31 & & 7 & & & & & & \\
\hline \multirow{2}{*}{\multicolumn{5}{|c|}{$\begin{array}{l}\text { Heterogeneity: } \chi^{2}=1.16, \mathrm{df}=1(P=0.28) ; I^{2}=14 \% \\
\text { Test for overall effect: } Z=2.53(P=0.01)\end{array}$}} & & 0.01 & 0.1 & 10 & 100 \\
\hline & & & & & & & \multicolumn{3}{|c|}{ Placebo Tofacitinib $10 \mathrm{mg}$} \\
\hline
\end{tabular}

(g) DAS 28-4 ESR $<2.6$ at month 3 (Tofacitinib $10 \mathrm{mg}$ )

\begin{tabular}{|c|c|c|c|c|c|c|c|c|}
\hline \multirow{2}{*}{ Study or subgroup } & \multicolumn{2}{|c|}{ facitinib $10 \mathrm{mg}$} & \multicolumn{2}{|c|}{ Placebo } & \multirow{2}{*}{ Weight } & \multirow{2}{*}{$\begin{array}{c}\text { Odds ratio } \\
\text { M-H, fixed, } 95 \% \text { CI }\end{array}$} & \multirow{2}{*}{\multicolumn{2}{|c|}{$\begin{array}{l}\text { Odds ratio } \\
\text { M-H, fixed, } 95 \% \text { CI }\end{array}$}} \\
\hline & Events & Total & Events & Total & & & & \\
\hline Kremer et al. (2011) & 40 & 270 & 4 & 148 & $56.5 \%$ & $6.26[2.19,17.87]$ & & - \\
\hline van der Heijde et al. (2013) & 41 & 257 & 2 & 129 & $28.7 \%$ & $12.05[2.87,50.68]$ & & \\
\hline van Vollenhoven et al. (2012 & 2) 22 & 176 & 1 & 92 & $14.8 \%$ & $13.00[1.72,98.07]$ & & 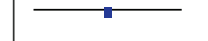 \\
\hline Total $(95 \% \mathrm{CI})$ & & 703 & & 369 & $100.0 \%$ & $8.92[4.10,19.42]$ & & \\
\hline Total events & 103 & & 7 & & & & & \\
\hline \multirow{2}{*}{\multicolumn{5}{|c|}{$\begin{array}{l}\text { Heterogeneity: } \chi^{2}=0.74, \mathrm{df}=2(P=0.69) ; I^{2}=0 \% \\
\text { Test for overall effect: } Z=5.51(P<0.00001)\end{array}$}} & & 0.01 & 0.1 & 100 \\
\hline & & & & & & \multicolumn{3}{|c|}{ Placebo Tofacitinib $10 \mathrm{mg}$} \\
\hline
\end{tabular}

(h) DAS 28-4 ESR $<2.6$ at month 6 (Tofacitinib $10 \mathrm{mg}$ )

FIGURE 1: Forest plots showing ACR 20 response and DAS 28-4 ESR $<2.6$ at month 3 and 6 with Tofacitinib 5 mg and 10 mg twice daily. 
TABLE 4: Revised ACR 1987 criteria for diagnosis of rheumatoid arthritis (RA).

\begin{tabular}{|c|c|}
\hline Criterion & Definition \\
\hline (1) Morning stiffness & Morning stiffness in and around the joints, lasting at least 1 hour before maximal improvement \\
\hline (2) Arthritis of 3 or more joint areas & $\begin{array}{l}\text { At least } 3 \text { joint areas simultaneously have had soft tissue swelling or fluid (not bony overgrowth } \\
\text { alone) observed by a physician. The } 14 \text { possible areas are right or left PIP, MCP, wrist, elbow, knee, } \\
\text { ankle, and MTP joints }\end{array}$ \\
\hline (3) Arthritis of hand joints & At least 1 area swollen (as defined above) in a wrist, MCP, or PIP joint \\
\hline (4) Symmetric arthritis & $\begin{array}{l}\text { Simultaneous involvement of the same joint areas (as defined in } 2 \text { ) on both sides of the body } \\
\text { (bilateral involvement of PIPs, MCPs, or MTPs is acceptable without absolute symmetry) }\end{array}$ \\
\hline (5) Rheumatoid nodules & $\begin{array}{l}\text { Subcutaneous nodules over bony prominences, extensor surfaces, or juxtaarticular regions, observed } \\
\text { by a physician }\end{array}$ \\
\hline (6) Serum rheumatoid factor & $\begin{array}{l}\text { Demonstration of abnormal amounts of serum rheumatoid factor by any method for which the } \\
\text { result has been positive in }<5 \% \text { of normal control subjects }\end{array}$ \\
\hline (7) Radiographic changes & $\begin{array}{l}\text { Radiographic changes typical of rheumatoid arthritis on posteroanterior hand and wrist } \\
\text { radiographs, which must include erosions or unequivocal bony decalcification localized in or most } \\
\text { marked adjacent to the involved joints (osteoarthritis changes alone do not qualify) }\end{array}$ \\
\hline
\end{tabular}

Note: criteria 1 through 4 must have been present for at least 6 weeks. Rheumatoid arthritis is defined by the presence of 4 or more criteria, and no further qualifications (classic, definite, or probable) or list of exclusions are required.

$(P=0.004)$ regarding anaemia (decrease in haemoglobin of -1 to $-3 \mathrm{~g} / \mathrm{dL}$ ) at month $0-3$ (Table 3 ).

Elevation of LDL cholesterol was reported to be significant in both Tofacitinib $5 \mathrm{mg}$ and Tofacitinib $10 \mathrm{mg}$ groups compared to placebo in two phase 3 trials $\{(P<0.001)[16]$ and $(P<0.0001)[15]\}$.

\section{Discussion}

Tofacitinib is the first orally active biologic agent approved by US FDA in November 2012 for use in moderate to severe active adult RA patients with prior inadequate response to, or who are intolerant of, methotrexate either as monotherapy or combined with methotrexate. Combination therapy with other biologics is not recommended [31]. It is a potent inhibitor of JAK 1 and JAK 3 which binds to $\beta$ and $\gamma$ chains of cytokine receptor, respectively, to which inflammatory mediators such as IL-2, IL-4, IL-7, IL-9, IL-15, and IL-21 attach to initiate synovial inflammation in RA $[32,33]$.

In this review, we assessed the efficacy and safety of Tofacitinib in active RA patients by analyzing the results of randomized placebo-controlled clinical trials. Ten studies are identified, eight of which are included in data analysis with a total of 4347 participants. The trials reviewed were of a similar design with at least three arms: placebo, Tofacitinib $5 \mathrm{mg}$ twice daily, and Tofacitinib $10 \mathrm{mg}$ twice daily with duration of 3 to 24 months. However, the results are only available till month 12. Longer clinical trials with results are required for full evaluation of long-term efficacy and safety of Tofacitinib.

There are three primary efficacy outcome measures of this review: ACR 20 response, DAS 28-4 ESR < 2.6, and improvement in HAQ-DI score. Two doses of Tofacitinib $5 \mathrm{mg}$ and $10 \mathrm{mg}$ twice daily produced statistically significant ACR 20 response at month 3 and month 6 compared to placebo $(P<$ $0.00001)$. For DAS $28-4 \mathrm{ESR}<2.6$, Tofacitinib $5 \mathrm{mg}$ did not result in significant change at month $3(P=0.13)$. However, at month $6,5 \mathrm{mg}$ twice daily dosage provided significant improvement $(P<0.0001)$. Higher dosage of $10 \mathrm{mg}$ twice daily showed significance since the early period of month 3 $(P=0.01)$. More significant improvement in DAS 28-4 ESR $<2.6$ achievement was noted at month $6(P<0.00001)$. Improvement in HAQ-DI score from the baseline was also consistently better for oral Tofacitinib $5 \mathrm{mg}$ and $10 \mathrm{mg}$ twice daily compared with placebo. These promising efficacy results combined with its oral route of administration would make Tofacitinib a better choice as a biologic for active RA patients not responding to methotrexate or other DMARDs.

Nevertheless, trials on head to head comparison between Tofacitinib and other approved biologic agents are lacking. Only one trial in this review included subcutaneous adalimumab 40 mg every 2 weeks as one arm in the study design [19]. Further randomized controlled trials comparing the efficacy of Tofacitinib with other approved biologic agents for RA are recommended.

Like other biologic agents, Tofacitinib has the risk of infections, cancer, lymphomas, and cytopenias. Active tuberculosis (TB) is one of the serious infections that usually occur with biologic agents, and US FDA recommends to rule out latent $\mathrm{TB}$ prior to initiation of therapy and to monitor for active TB during treatment period [34]. In this meta-analysis, the risk of serious adverse events and serious infections with Tofacitinib $5 \mathrm{mg}$ and $10 \mathrm{mg}$ twice daily was not significantly higher in relation to the placebo group at month 3. Similarly, these safety outcome measures are not statistically different among $5 \mathrm{mg}$ and $10 \mathrm{mg}$ dosages at month 6 of therapy. Common adverse events reported in individual phase 3 studies were infections, headache, nausea, vomiting, and diarrhea. Infections included bronchitis, nasopharyngitis, upper respiratory tract infection, pneumonia, cellulitis, urinary tract infection, and herpes zoster [35].

Cytopenias, especially decrease in neutrophil count and haemoglobin, were reported among Tofacitinib-treated RA patients. Occurrence of mild neutropenia (1500-1999 cells $/ \mathrm{mm}^{3}$ ) was higher than moderate to severe neutropenia 
(500-1499 cells $/ \mathrm{mm}^{3}$ ) with Tofacitinib group, which was not statistically significant in comparison with placebo group on meta-analysis of the studies. There were no reported cases of life-threatening neutropenia across the studies. Reduction in haemoglobin by $1-3 \mathrm{~g} / \mathrm{dL}$ from the baseline is expected to be higher among Tofacitinib group compared to placebo. However, meta-analysis of the data across the included studies revealed an interesting result of protective effect on anaemia by Tofacitinib, compared to placebo group with $5 \mathrm{mg}$ dosage $(P=0.004)$. Detailed observation of the placebo group was needed before we conclude the protective effect of Tofacitinib $5 \mathrm{mg}$ in anaemia. Nonetheless, it can be confidently concluded that Tofacitinib does not increase the risk of anaemia with both $5 \mathrm{mg}$ and $10 \mathrm{mg}$ doses. Lesser inhibition of JAK 2, which is responsible for erythropoiesis, by Tofacitinib might explain this finding.

Other reported side effects of Tofacitinib include hypercholesterolaemia and rise in liver enzymes (which rarely exceeds beyond 3 times upper limit) and serum creatinine. In a meta-analysis of safety profile of protein kinase inhibitors, hypercholesterolaemia was reported to be significantly higher with Tofacitinib than the comparator group with dose-related increase in mean serum total cholesterol, HDL, and LDL at week 6 [36]. Nevertheless, larger trials with longer duration of study and postmarketing adverse event reporting are necessary for monitoring of long-term safety of this new effective antirheumatic drug.

\section{Conclusion}

In summary, Tofacitinib is an effective oral biologic agent with manageable safety profile for active RA patients with incomplete response to other DMARDs.

$\begin{array}{ll}\text { Abbreviations } \\ \text { ACR: } & \text { American college of rheumatology } \\ \text { CDC: } & \text { Center for disease control } \\ \text { CRP: } & \text { C-reactive protein } \\ \text { DAS: } & \text { Disease activity score } \\ \text { DMARD: } & \text { Disease modifying antirheumatic drug } \\ \text { ESR: } & \text { Erythrocyte sedimentation rate } \\ \text { HAQ-DI: } & \text { Health Assessment } \\ & \text { Questionnaire-Disability Index } \\ \text { HLA: } & \text { Human leucocyte antigen } \\ \text { IL: } & \text { Interleukin } \\ \text { JAK: } & \text { Janus kinase } \\ \text { NSAID: } & \text { Nonsteroidal anti-inflammatory drug } \\ \text { RA: } & \text { Rheumatoid arthritis } \\ \text { STAT: } & \text { Signal transducer and activator of } \\ & \text { transcription } \\ \text { TB: } & \text { Tuberculosis } \\ \text { TNF: } & \text { Tumour necrosis factor } \\ \text { US FDA: } & \text { United States Food and Drug } \\ & \text { Administration } \\ \text { WHO: } & \text { World Health Organization } \\ \text { YLD: } & \text { Years lived with disability. }\end{array}$

\section{Conflict of Interests}

The authors declare that there is no conflict of interests.

\section{References}

[1] P. M. Brooks, "The burden of musculoskeletal disease-a global perspective," Clinical Rheumatology, vol. 25, no. 6, pp. 778-781, 2006.

[2] A. J. Silmanand and J. E. Pearson, "Epidemiology and genetics of rheumatoid arthritis," Arthritis Research, vol. 4, supplement 3, pp. S265-S272, 2002.

[3] "Rheumatoid Arthritis: centers for disease control and prevention," http://www.cdc.gov/arthritis/basics/rheumatoid.htm.

[4] D. Symmons, C. Mathers, and B. Pfleger, "The global burden of rheumatoid arthritis in the year 2000," Working Paper, World Health Organization, Geneva, Switzerland, http://www .who.int/healthinfo/statistics/bod_rheumatoidarthritis.pdf.

[5] C. G. Helmick, D. T. Felson, R. C. Lawrence et al., "Estimates of the prevalence of arthritis and other rheumatic conditions in the United States. Part I," Arthritis and Rheumatism, vol. 58, no. 1, pp. 15-25, 2008.

[6] T. Pincus, Y. Yazici, T. Sokka, D. Aletaha, and J. S. Smolen, "Methotrexate as the "anchor drug" for the treatment of early rheumatoid arthritis," Clinical and Experimental Rheumatology, vol. 21, no. 5, supplement 31, pp. S179-S185, 2003.

[7] V. C. Kyttaris, "Kinase inhibitors: a new class of antirheumatic drugs," Drug Design, Development and Therapy, vol. 6, pp. 245250, 2012.

[8] L.-D. Quan, G. M. Thiele, J. Tian, and D. Wang, "The development of novel therapies for rheumatoid arthritis," Expert Opinion on Therapeutic Patents, vol. 18, no. 7, pp. 723-738, 2008.

[9] J. S. Smolen, R. Landewé, F. C. Breedveld et al., "EULAR recommendations for the management of rheumatoid arthritis with synthetic and biological disease-modifying antirheumatic drugs," Annals of the Rheumatic Diseases, vol. 69, no. 6, pp. 964975, 2010.

[10] J. A. Singh, D. E. Furst, A. Bharat et al., "2012 update of the 2008 American college of rheumatology recommendations for the use of disease-modifying antirheumatic drugs and biologic agents in the treatment of rheumatoid arthritis," Arthritis Care and Research, vol. 64, no. 5, pp. 635-639, 2012.

[11] I. B. McInnes and G. Schett, "The pathogenesis of rheumatoid arthritis," The New England Journal of Medicine, vol. 365, no. 23, pp. 2205-2219, 2011.

[12] P. Emery and T. Dörner, "Optimising treatment in rheumatoid arthritis: a review of potential biological markers of response," Annals of the Rheumatic Diseases, vol. 70, no. 12, pp. 2063-2070, 2011.

[13] Y. Yazici and A. L. Regens, "Promising new treatments for rheumatoid arthritis: the kinase inhibitors," Bulletin of the NYU Hospital for Joint Diseases, vol. 69, no. 3, pp. 233-237, 2011.

[14] S. H. Park, S. K. Kim, and J. Y. Choe, "Biologic agent for rheumatoid arthritis," Hanyang Medical Reviews, vol. 32, no. 2, pp. 68-76, 2012.

[15] G. R. Burmester, R. Blanco, C. Charles-Schoeman et al., "Tofacitinib (CP-690, 550) in combination with methotrexate in patients with active rheumatoid arthritis with an inadequate response to tumour necrosis factor inhibitors: a randomised phase 3 trial," The Lancet, vol. 381, no. 9865, pp. 451-460, 2013. 
[16] R. Fleischmann, J. Kremer, J. Cush et al., "Placebo-controlled trial of tofacitinib monotherapy in rheumatoid arthritis," The New England Journal of Medicine, vol. 367, no. 6, pp. 495-507, 2012.

[17] J. Kremer, Z.-G. Li, S. Hall et al., “Tofacitinib (CP-690, 550), an oral JAK inhibitor, in combination with traditional DMARDs: phase 3 study in patients with active rheumatoid arthritis with inadequate response to DMARD," Annals of the Rheumatic Diseases, vol. 70, supplement 3, p. 170, 2011, Late breaking abstracts at EULAR.

[18] D. van der Heijde, Y. Tanaka, R. Fleischmann et al., "Tofacitinib (CP-690, 550) in patients with rheumatoid arthritis receiving methotrexate: twelve-month data from a twenty-four-month phase III randomized radiographic study," Arthritis \& Rheumatism, vol. 65, no. 3, pp. 559-570, 2013.

[19] R. F. van Vollenhoven, R. Fleischmann, S. Cohen et al., "Tofacitinib or adalimumab versus placebo in rheumatoid arthritis," The New England Journal of Medicine, vol. 367, no. 6, pp. 508519, 2012.

[20] R. Fleischmann, M. Cutolo, M. C. Genovese et al., "Phase IIb dose-ranging study of the oral JAK inhibitor tofacitinib (CP-690,550) or adalimumab monotherapy versus placebo in patients with active rheumatoid arthritis with an inadequate response to disease-modifying antirheumatic drugs," Arthritis and Rheumatism, vol. 64, no. 3, pp. 617-629, 2012.

[21] J. M. Kremer, S. Cohen, B. E. Wilkinson et al., "A phase IIb doseranging study of the oral JAK inhibitor tofacitinib (CP-690, 550) versus placebo in combination with background methotrexate in patients with active rheumatoid arthritis and an inadequate response to methotrexate alone," Arthritis \& Rheumatism, vol. 64, no. 4, pp. 970-981, 2012.

[22] Y. Tanaka, M. Suzuki, H. Nakamura, S. Toyoizumi, and S. H. Zwillich, "Phase II study of tofacitinib (CP-690,550) combined with methotrexate in patients with rheumatoid arthritis and an inadequate response to methotrexate," Arthritis Care and Research, vol. 63, no. 8, pp. 1150-1158, 2011.

[23] J. M. Kremer, B. J. Bloom, F. C. Breedveld et al., "The safety and efficacy of a JAK inhibitor in patients with active rheumatoid arthritis: results of a double-blind, placebo-controlled phase IIa trial of three dosage levels of CP-690,550 versus placebo," Arthritis and Rheumatism, vol. 60, no. 7, pp. 1895-1905, 2009.

[24] J. H. Coombs, B. J. Bloom, F. C. Breedveld et al., "Improved pain, physical functioning and health status in patients with rheumatoid arthritis treated with CP-690,550, an orally active Janus kinase (JAK) inhibitor: results from a randomised, double-blind, placebo-controlled trial," Annals of the Rheumatic Diseases, vol. 69, no. 2, pp. 413-416, 2010.

[25] G. R. Stark and J. E. Darnell Jr., "The JAK-STAT pathway at twenty," Immunity, vol. 36, no. 4, pp. 503-514, 2012.

[26] J. J. O'Shea, S. M. Hollandand, and L. M. Staudt, "JAKs and STATs in immunity, immunodeficiency and cancer," The New England Journal of Medicine, vol. 368, pp. 161-170, 2013.

[27] J. J. O'Shea and R. Plenge, "JAK and STAT signaling molecules in immunoregulation and immune-mediated disease," Immunity, vol. 36, no. 4, pp. 542-550, 2012.

[28] D. L. Simmons, "Targeting kinases: a new approach to treating inflammatory rheumatic diseases," Current Opinion in Pharmacology, vol. 13, pp. 1-9, 2013.

[29] R. Fleischmann, "Novel small-molecular therapeutics for rheumatoid arthritis," Current Opininion in Rheumatology, vol. 24, pp. 335-341, 2012.
[30] C. Kahn, “Tofacitinib for Rheumatoid Arthritis", 2012, http:// www.fda.gov/downloads/AdvisoryCommittees/Committees MeetingMaterials/Drugs/ArthritisAdvisoryCommittee/ UCM304200.pdf.

[31] "FDA approves Xeljanz for rheumatoid arthritis," http://www .fda.gov/NewsEvents/Newsroom/PressAnnouncements/ ucm327152.htm.

[32] C. A. F. Zerbini and A. B. V. Lomonte, "Tofacitinib for the treatment of rheumatoid arthritis," Expert Review of Clinical Immunology, vol. 8, no. 4, pp. 319-331, 2012.

[33] K. Maeshima, K. Yamaoka, S. Kubo et al., "The JAK inhibitor tofacitinib regulates synovitis through inhibition of interferon$\gamma$ and interleukin-17 production by human CD4+ T cells," Arthritis \& Rheumatism, vol. 64, no. 6, pp. 1790-1798, 2012.

[34] Pfizer, "Tofacitinib for the Treatment of Rheumatoid Arthritis. NDA, 203214, Briefing Document for the May 9, 2012, meeting of the Arthritis Advisory Committee," http://www.fda.gov/ downloads/AdvisoryCommittees/CommitteesMeeting Materials/Drugs/ArthritisAdvisoryCommittee/UCM302960.

[35] K. M. O'Dell and A. E. Rummel, “Tofacitinib: a novel oral Janus kinase inhibitor for rheumatoid arthritis," Formulary, vol. 47, pp. 350-358, 2012.

[36] E. Salgado, J. R. Maneiro, L. Carmona, and J. J. GomezReino, "Safety profile of protein kinase inhibitors in rheumatoid arthritis: systematic review and meta-analysis," Annals of the Rheumatic Diseases, 2013. 


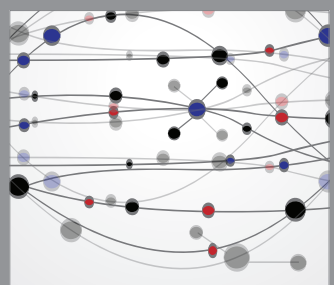

The Scientific World Journal
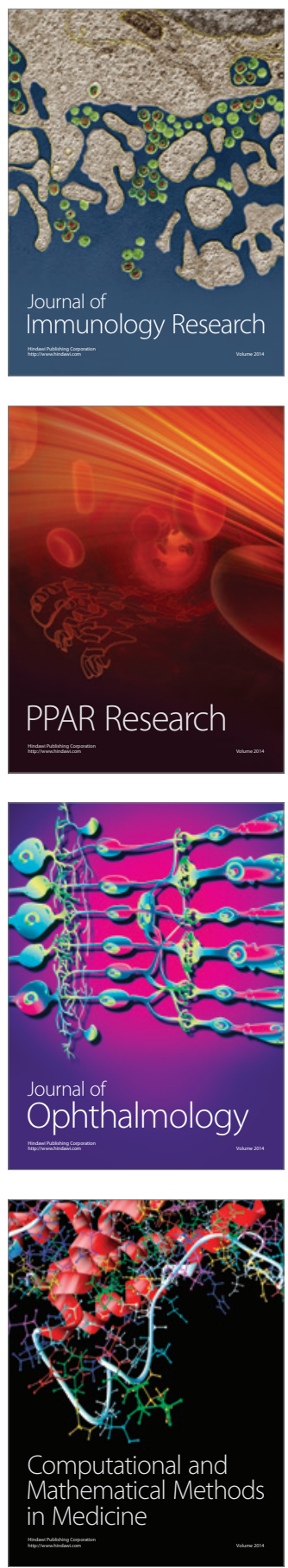

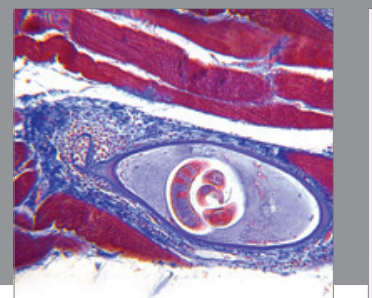

Gastroenterology

Research and Practice
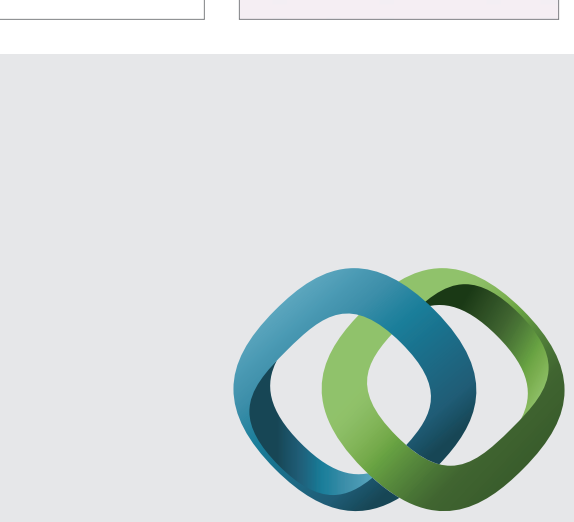

\section{Hindawi}

Submit your manuscripts at

http://www.hindawi.com
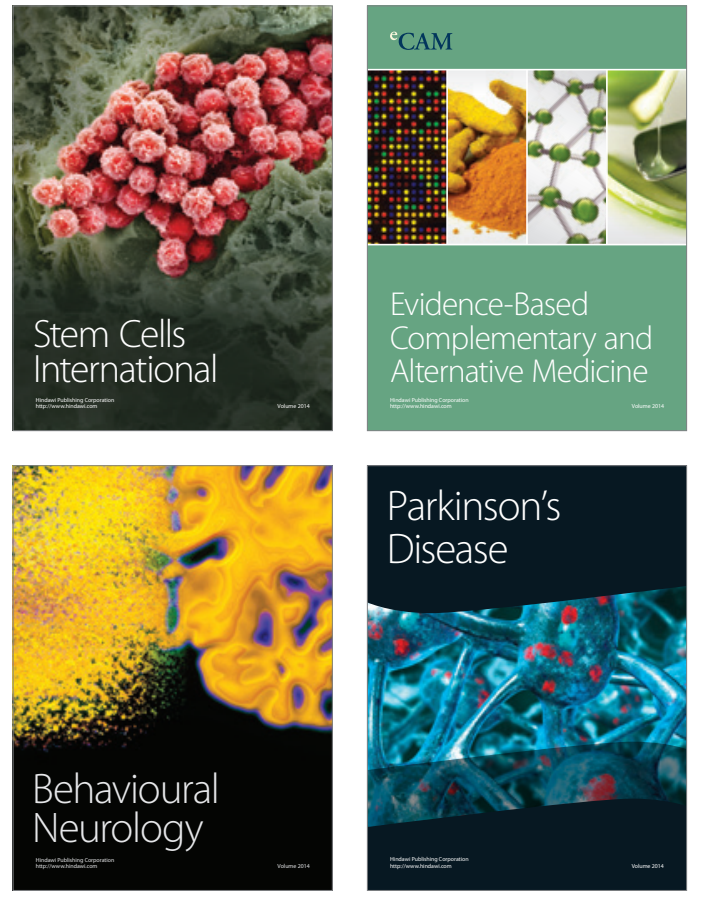
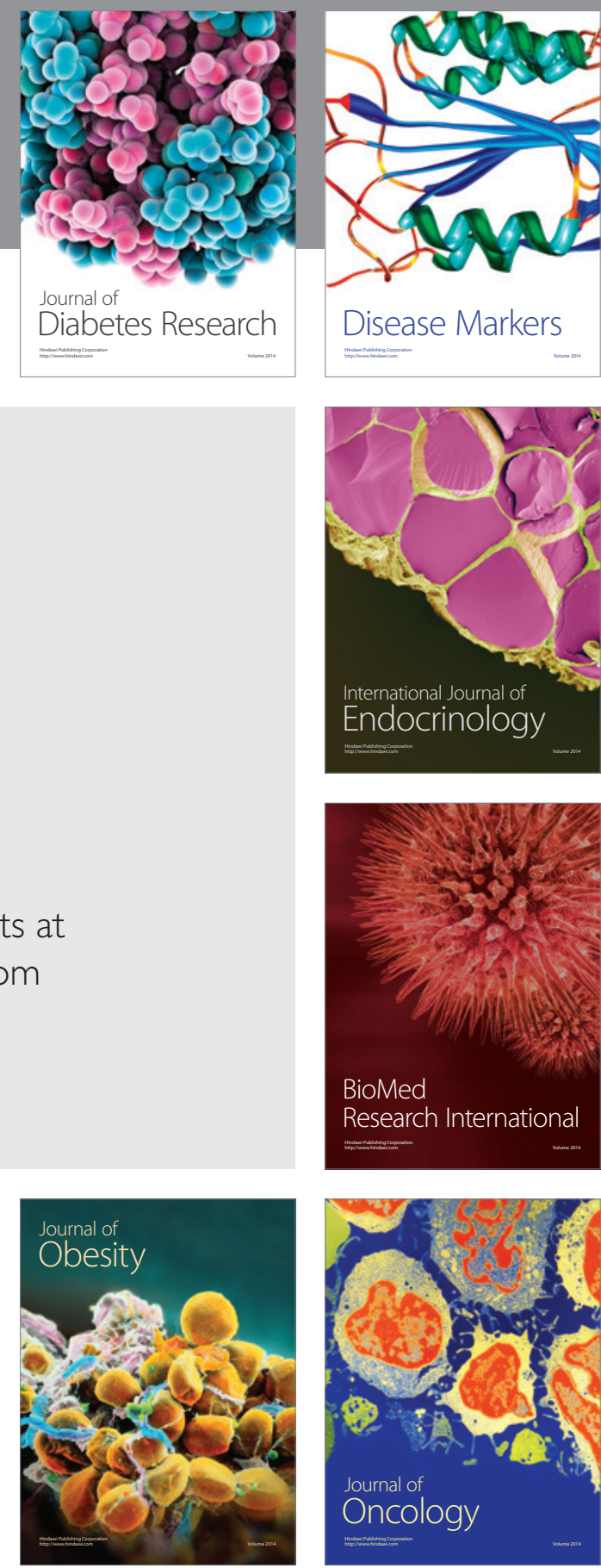

Disease Markers
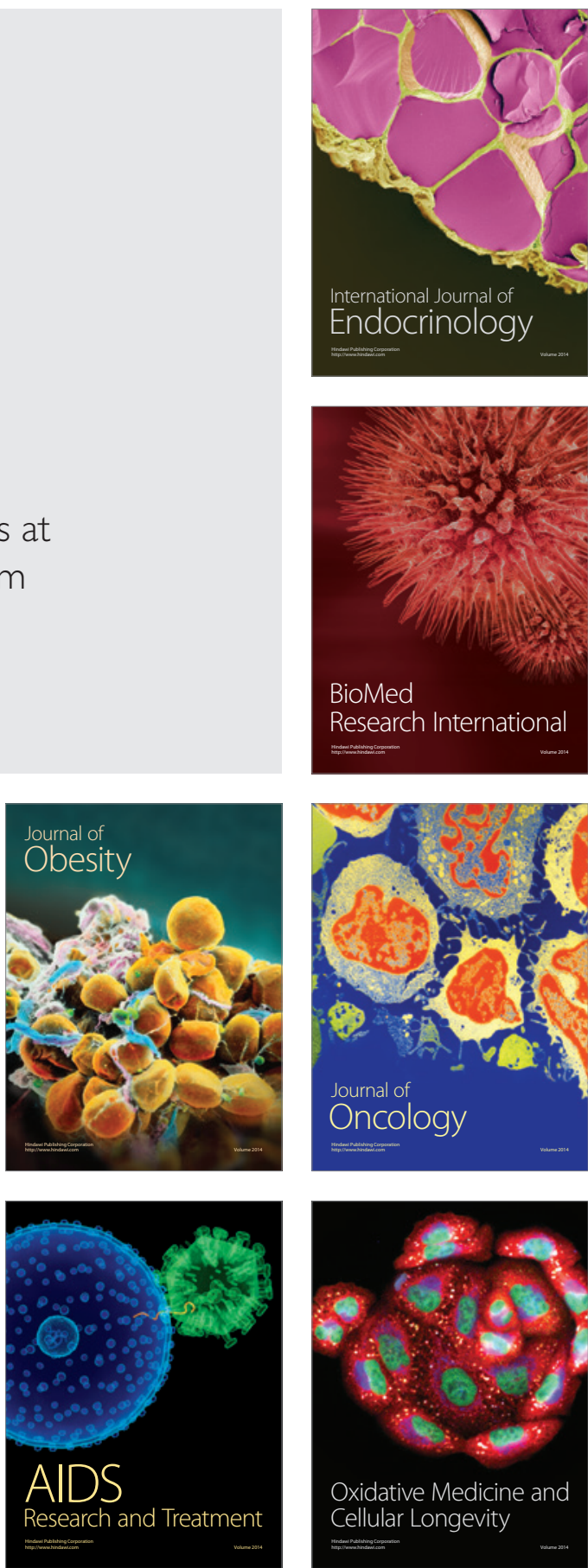